\title{
Conceptual Relation Between Quality of Life and Environmentally Friendly Food
}

\section{A S Khamitsaeva ${ }^{1}$, R I Osikina², E S Dzodzieva ${ }^{3}$, Z A Kubatieva ${ }^{3}$, F I Budaev ${ }^{4}$, V Kh Sebetov ${ }^{3}$, Z A Khortiev ${ }^{4}$, and A R Budaev ${ }^{4}$}

${ }^{1}$ Mountain State Agrarian University, Vladikavkaz, Russia

${ }^{2}$ North Caucasus Mining and Metallurgical Institute, Vladikavkaz, Russia

${ }^{3}$ Mountain State Agrarian University, Vladikavkaz, Russia

${ }^{4}$ North Ossetian State University named after K.L. Khetagurov, Vladikavkaz, Russia

Corresponding Author:

A S Khamitsaeva

allahamicaeva@mail.ru

Received: 25 October 2019

Accepted: 15 November 2019

Published: 25 November 2019

Publishing services provided by

Knowledge E

(c) A S Khamitsaeva et al. This article is distributed under the terms of the Creative Commons

Attribution License, which

permits unrestricted use and redistribution provided that the original author and source are credited.

Selection and Peer-review under the responsibility of the AgroSMART 2019 Conference Committee.

\section{Abstract}

One of the most important indicators of the quality of life is environmentally friendly nutrition implying the use and rational combination of eco-friendly raw materials contained in recipes of new generation products, thus guaranteeing full supply of food and biologically active agents to all vital systems of an organism. The development of the society fosters the increasing interdependence and interconditionality of processes within the "nature-person-society" system. The current environmental situation brought the problem of environmental culture and its role in the optimization of socionatural interaction to the foreground. The paper presents the materials of theoretical justification of conceptual relations between the quality of life and eco-friendly nutrition; it describes pilot studies of safety indicators, polysaccharide composition of aromatic plants for their use in the production of functional food to enrich the composition with protective components. It is established that the studied aromatic plants (AP) growing in environmentally friendly territories of the Republic of North Ossetia-Alania are characterized by low weight ratio of toxic substances (below admissible hygienic standards), high content of dietary fibers (DF). The sorption capacity of AP powders in relation to lead is studied. All studied powders of AP modification products are characterized by high sorption capacity. The technologies of flour food with addition of AP powders are developed. It is revealed that in comparison with control samples certain flour food products almost twice reduce the lead content in the blood of laboratory infant rats.

Keywords: aromatic plants, quality of life, sorption capacity, dietary fibers, foodstuff, ecological recurrence

\section{Introduction}

The purpose of the study is to expand the use of aromatic plants in functional food technology, which is not only technologically important, but also bears a great social value in regulating the relation between the quality of life and healthy nutrition. It is possible to prevent diseases caused by harmful environmental impurities by consuming enriched products, including dietary fibers of aromatic plants. 
The tasks of the study are as follows:

1. to provide theoretical justification of conceptual relation between the quality of life and healthy nutrition;

2. to prove the choice of aromatic plants, to estimate their potential;

3. to study the safety indicators of aromatic plants;

4. to define the sorption capacity of aromatic plants;

5. to study the sorption capacity of developed flour products on laboratory animals.

\section{Methods and Materials}

The following powders of dried up plants are chosen for the study: ramson, thyme, dill seeds, lemon balm, greater nettle, as well as finished flour products. The safety of raw materials and finished goods was defined by the content of potentially dangerous chemicals and microbiological indicators specified in SanPiN 2.3.2.1078-01 [6]. Toxic elements were defined via atomic absorption spectrometry according to GOST 2693286; radionuclide $\mathrm{Sr}^{90}$ was defined in accordance with GOST 32163-2013. Foodstuff. Method for strontium Sr-90 content determination; mycotoxins in accordance with GOST P 50474-93. Foodstuff. Methods for detection and quantity determination of coliformes.

The binding ability of lead ions with dietary fibers was estimated via the technique modified by Tamova M.Yu. The experimental biomaterials were studied on automatic analyzer ARCHITECT 8000 using standard methods.

The appeal to environmental aspect of the quality of life is caused by the fact that the environmental situation does not only serve a prerequisite to improve the quality of life, but also its major component. The relevance of the study is bound to increased "concern of environmental safety since environmental changes may affect health, and at times may even limit the survival capability", as stated in the report on Caring for the Future by the Independent Committee on Problems of the Population and Quality of Life [2, 3].

The quality of life has no conventional formalized structure and a standard set of indicators. The priorities depend on human needs and on the development level of countries and regions, therefore the assessment criteria for developed and developing countries do not coincide. The assessment of the quality of life is quite difficult on the one hand due to multidimensionality of this concept, and on the other hand due to the fact that different population groups understand the quality of life differently, and hence 
many domestic and foreign researchers propose subjective methods of assessment $[4,5,13]$.

In Russia the quality of life is closely connected with the standard of living and is expressed by the following quantifying indicators: cash income on average per capita a month, population with cash income below poverty line, per capita consumption of basic foodstuffs, quality of these products, environmental safety of food, structure and culture of consumption [4, 13].

In recent years Russia is obviously facing negative trends in demographic processes: average life expectancy is reducing, mortality and morbidity rates are growing. The main morbidity reasons are the following: unhealthy food caused by the consumption of harmful foodstuff; oncological, gastrointestinal diseases are inter alia caused by environmental recurrence $[3,4,13,14]$.

Thus, one of the priority directions of the state policy in improving the quality of life is to create technologies of qualitatively new types of foodstuff preventing various diseases, strengthening protective functions of an organism and ensuring adequate adaptation of a person to the environment.

The difficulties related to the range of functional food are caused by the lack of system conceptual developments and the legislative base to regulate the organization of functional food, to assess and control its quality. Further achievements in the food theory in relation to human activity in the conditions of environmental crisis and social tension made it possible to conclude that the prevention of diseases and fight for active longevity shall consider individual variability of people. It is impossible to create diets optimally aimed to prevent all diseases, but it is possible to ensure the alimentary impact on primary diseases [10--12].

At the turn of the $21^{\text {st }}$ century the academician of the Russian Academy of Medical Science V.A. Tutelyan introduced the concept of "optimum nutrition" of the Russian population. This scientific concept incorporated almost all achievements of modern science of nutrition in Russia taking into account the existing environmental situation and the increasing influence of technogenic burden against the background of damaged food patterns in the country. Considering the obvious deficiency of macro- and micronutrients, V.A. Tutelyan believes that the health promotion of the population is only possible through the integrated approach to the optimum nutrition. First of all, this includes the supply of primary nutrition of a person with natural and high-quality food. Other elements of the optimum nutrition are aimed to enrich the composition of foodstuff with scarce nutrients considering the widespread introduction of biologically 
active, not expensive environmentally friendly vegetable ingredients of domestic origin [10--13].

Flour industry plays the major role in the development of foodstuff with increased nutrition value and expansion of the nutrition range since its products are daily consumed by all population groups in the Russian Federation. According to the key selection principles of vegetable raw materials in the technology of flour functional products the following were considered: biological value, potential, technological properties of raw materials ensuring preventive action of some foodstuff. The use of aromatic plants rich with biologically active agents is quite efficient and expedient from scientific, technological, and environmental perspectives improving the quality of foodstuff.

It is not fortuitous that among critical technologies of the priority fields of science and technology the governmental commission on scientific and technical policy referred to the systems of life support and protection of a person in extreme conditions, biological food, safety of foodstuff [11--13].

According to the theory of adequate food proposed by the authors (Vernadsky V.I. and Ugolev A.M.) against the background of the reducing human health it is possible to improve the internal environment by introducing such biological factors as protective, antioxidant, vitamin, macro- and microelement, etc. into foodstuff [1, 13].

The subject of the specified theory includes common patterns to assimilate vital substances at all levels of the living matter, while its applied tasks include the problem of optimum nutrition taking into account natural and production technologies in the conditions of environmental recurrence and economic crisis.

Most likely, healthy products will ensure general and nonspecific action on an organism and will serve an intermediate link between the groups of traditional foodstuff and medicines $[5,9,13]$.

The specified concept confirms close interaction between the structure of food of a person and the state of his health.

Poor, unbalanced, low-quality nutrition inevitably leads to health deterioration of the nation. It absorbed almost all achievements of modern science of nutrition in Russia taking into account the current environmental situation and the increasing impact of harmful nutritive factors $[7,8,10,14]$.

Preventive methods that would decrease the accumulation of heavy metals in an organism and reduce thus caused pathological changes are connected with the development of new functional food. For this purpose, the preventive ability of powders of dried aromatic plants (AP) for their further use in the recipes of functional flour 
finished products was studied and preclinical trials proving protective properties of the developed bakery products were conducted.

\section{Results}

The safety indicators of initial raw materials were analyzed to receive environmentally friendly products. For comparison of AP safety indicators, the study shows the content of toxic elements, mycotoxins, radionuclides according to SanPiN requirements 2.3.2 1078-01. The study showed that the weight ratio of AP toxic substances is below the admissible hygienic standards that makes it possible to recommend them as eco-friendly raw materials for functional flour products. The adequate content of toxic substances is typical for plants growing in mountain and foothill environmentally safe territories of the Republic of North Ossetia-Alania.

The polysaccharides of aromatic plants are efficient gastrointestinal adsorbents able to connect and remove heavy-metal ions and radionuclides from an organism. Besides, they have high potential to prevent and treat gastrointestinal and cardiovascular diseases. In view of the above we studied the content of dietary fibers in modified aromatic plants (AP) (Tabl. 1).

Aromatic plants (ramson, lemon balm, greater nettle, thyme, dill seeds) are characterized by high content of dietary fibers (DF).

The sorption capacity of AP powders in relation to lead was studied. The choice out of this toxic element is caused by the fact that according to Rospotrebnadzor of the Republic of North Ossetia-Alania, its content in air exceeds the maximum allowable concentration.

All studied powders of AP modification products are characterized by high sorption capacity. The sequence of AP activity in ascending order by sorption capacity of lead is established: greater nettle $\rightarrow$ lemon balm $\rightarrow$ ramson $\rightarrow$ dill seeds $\rightarrow$ thyme (Fig. 1).

TABLE 1: Content is dietary fibers in AP.

\begin{tabular}{|l|c|c|c|c|c|}
\hline & \multicolumn{5}{|c|}{ Weight ratio, \% } \\
\hline & Ramson & Thyme & Dill seeds & Lemon balm & Greater nettle \\
\hline Fibre & 2.8 & 1.8 & 2.3 & 2.1 & 2.7 \\
\hline Pectin & 1.7 & 2.3 & 2.6 & 1.2 & 1.3 \\
\hline
\end{tabular}

The obtained results open prospects for using them in the technology of functional flour products with high sorption properties. 


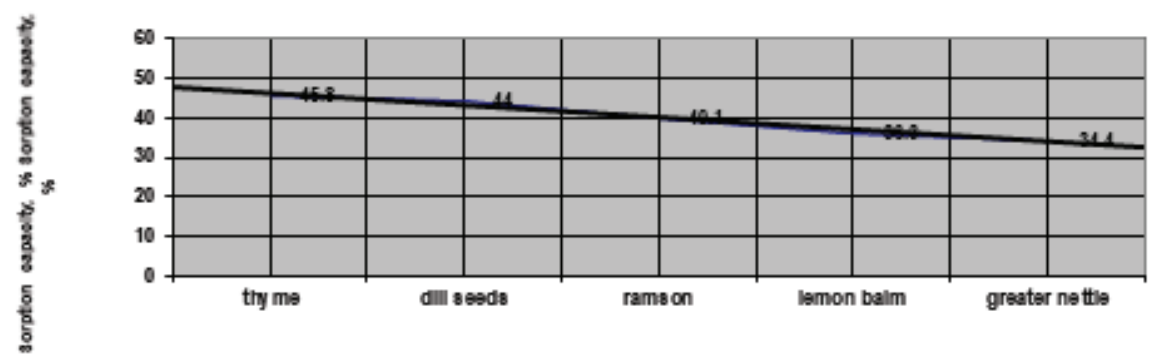

Figure 1: Sorption capacity of aromatic plant powders in relation to lead.

TABLE 2: Dynamics of lead content in blood serum of experimental animals.

Flour products
Control
Medical
Improved, Elkhotovsky

$\mathrm{Pb}$ content in blood, $\mathrm{mg} / \mathrm{l}$
at the beginning of the
experiment
$2.63 \pm 0.016$
$2.96 \pm 0.015$
$3.01 \pm 0.026$

$\mathrm{Pb}$ content in blood, $\mathrm{mg} / \mathrm{l}$ at
the end of the experiment
$2.50 \pm 0.09$
$1.99 \pm 0.013$
$1.87 \pm 0.011$

The experiments to determine the sorption capacity of developed functional flour products were carried out on laboratory animals. Table 2 shows the results of the study.

Systematic four-week feeding of experimental animals showed that in comparison with control samples certain flour food products almost twice reduce the lead content in the blood of laboratory infant rats. The results confirmed preventive, medical sorbing effect of developed flour products with AP powders.

The main sorption mechanism is the ability to accumulate and reduce the toxic effect of harmful pectin substances, as well as complex formation caused by high electron donor ability of atoms of oxygen, nitrogen and others. Considering the known bioprotective and enterosorbing properties of dietary fibers (DF) it is possible to confirm our assumption on increased physiological value of developed functional products by enriching their recipes with biologically active AP agents that contain the standard quantity of dietary fibers and correspond to preventive effect.

\section{Conclusion}

Thus, based on the theoretical study of the quality of life and safe nutrition as ideal and interpenetrating aspects we analyzed the role of environmental factor in optimizing the socioenvironmental interaction and relation. Against the background of the reducing human health it is possible to improve the quality of life within the existing conditions of 
environmental recurrence and economic crisis by enriching food with such biological factors as protective, macro- and microelement, etc.

Due to their biochemical properties the use of aromatic plants, such as greater nettle, lemon balm, ramson, dill seeds, and thyme in the development of functional flour products is quite relevant to regulate the polysaccharide structure of the final product thus realizing their biological potential to improve the revitalizing indicator of the nation.

The specified vegetable ingredients can be considered as a prominent resource within the food complex of the Republic of North Ossetia-Alania, which does not require huge investments for their wide and rational use.

\section{References}

[1] Vernadsky, V.I. (1972). Biosphere, ed. M.S. Gilyarov. Moscow: Mir, pp. 8--13.

[2] Caring for the future. (1998). Report of the Independent Committee on Problems of the Population and Quality of Life. Moscow, pp. 6--8.

[3] Zakharova, E.Yu. (2011). Ecological culture of social and natural ecosystem. Chita: Publishing house of Transbaikal State University, pp. 2--3.

[4] Lega, M.B., Shchetkina, I.A. (2011). Quality of life: organizational and administrative aspect. Novosibirsk: Nauka, pp. 5--6.

[5] Markovich, D.G., Zhukov, V.I., Bganba-Cerera, V.R. (1998). Social ecology. Moscow: Soyuz publishing house of MSUCE, pp. 16--48.

[6] SanPiN 2.3.2.1078-01. (2002) Hygienic requirements of safety and nutrition value of foodstuff: [approved by the chief public health physician of the Russian Federation on 06.10.2001: introduced on 01.09.2002]. Moscow: Russian Ministry of Health, pp. 165.

[7] Strupan, E.A., Kolodyaznaya, V.S., Strupan, O.A. (2012) Conversion technology of wild-growing raw materials. Bulletin of Krasnoyarsk State Agricultural University, no. 7, pp. 161--165.

[8] Sadovoy, V.V., Trubina, I.A., Shchedrina, T.V., Skorbina, E.A. (2017). Develop prescription food compositions intended for individual meals, Research Journal of Pharmaceutical. Biological and Chemical Sciences, vol. 8, no. 1, pp. 8925--8929.

[9] Sadovoy, V.V., Shchedrina, T.V., Melentyeva, V.V., Khamitsaeva, A.S. (2018). Forecasting the molecular properties of dietary supplement used in the recipe of foodstuff for diabetes mellitus prevention. Research Journal of Pharmaceutical, Biological and Chemical Sciences, vol. 9, no. 4, pp. 1620--1625. 
[10] Tutelyan, V.A., Knyazhev, V.A. (2000). Implementation of the state policy of healthy nutrition of the Russian population: scientific support. Questions of nutrition, no. 3, pp. 4--7.

[11] Federal and regional aspects of state policy in the field of healthy food. (2002). Proceedings of the international symposium. Kemerovo, pp. 22--39.

[12] Ugolev, A.M. (1991). Theory of adequate nutrition and trophology. Moscow: Nauka, pp. 8--12.

[13] Khamitsaeva, A.S. (2011). Development of theoretical bases and technologies of flour and meat products using modification products of vegetable feedstock. $\mathrm{PhD}$ dissertation. St. Petersburg, pp. 71--77.

[14] Yakovlev, S.A. (2006). Health promotion and prevention of premature aging by natural means. Moscow: Rosa, pp. 5--20. 